Rocha Paulinha, P., Reuss Strenzel, GM. (2020). Um modelo empírico para estimativa do Total de Sólidos em Suspensão empregando o sensor OLI na costa de Caravelas, Bahia, Brasil. GeoFocus, Revista Internacional de Ciencia y Tecnología de la Información Geográfica, 26, 121-137. http://dx.doi.org/10.21138/GF.674

\title{
UM MODELO EMPÍRICO PARA ESTIMATIVA DO TOTAL DE SÓLIDOS EM SUSPENSÃO EMPREGANDO O SENSOR OLI NA COSTA DE CARAVELAS, BAHIA, BRASIL
}

\author{
aPAULA ROCHA PAULINHA E ${ }^{\mathrm{b}}$ GIL MARCELO REUSS STRENZEL \\ Universidade Estadual de Santa Cruz \\ Campus Soane Nazaré de Andrade, Rod. Jorge Amado, km 16 - Salobrinho, Ilhéus - BA, 45662 - \\ 900, Brazil \\ ageo.paularocha@gmail.com, ${ }^{\text {bgmreuss@gmail.com }}$
}

\section{RESUMO}

Este estudo visa contribuir com o entendimento da dinâmica do Total de Sólidos em Suspensão (TSS) na costa de Caravelas, Extremo Sul da Bahia, Brasil. Imagens obtidas pelo sensor OLI (Landsat 8) em 29/12/2014 e 30/01/2015 foram usadas na criação de um modelo empírico preditivo do TSS superficial, elaborado por regressão linear ajustada por mínimos quadrados. Os resultados mostraram um bom ajuste para o dia 29/12/2014 $\left(\mathrm{RMSE}=5,89 ; \mathrm{RE}=19,2 \% ; \mathrm{R}^{2}=0,89\right.$; $\mathrm{N}=14)$ e um ajuste razoável para a imagem de 30/01/2015 $\left(\mathrm{RMSE}=13,33 ; \mathrm{RE}=57 \% ; \mathrm{R}^{2}=0,5\right.$; $\mathrm{N}=6$ ). O modelo mostrou-se consistente. Porém, o monitoramento dos impactos decorrentes das atividades dragagem seria mais efetivo após nova saída de campo delineada para considerar a variabilidade regional do TSS, incluindo a pluma gerada pela dragagem.

Palavras-chave: Parque Nacional Marinho de Abrolhos; Landsat 8; recifes de coral; cor do océano: impactos.

\section{AN EMPIRICAL MODEL FOR ESTIMATING TOTAL SUSPENDED SOLIDS USING THE SENSOR OLI ON THE COAST OF CARAVELAS, BAHIA, BRAZIL}

\section{ABSTRACT}

This study contributes to the understanding of the dynamics of Total Solids in Suspension (TSS) on the coast of Caravelas, Extreme South of Bahia, Brazil. Images obtained by the OLI sensor (Landsat 8) on $12 / 29 / 2014$ and $1 / 30 / 2015$ were used to the creation of an empirical predictive model of superficial TSS, elaborated by linear regression adjusted by least squares. The results showed a 


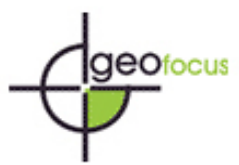

Rocha Paulinha, P., Reuss Strenzel, GM. (2020). Um modelo empírico para estimativa do Total de Sólidos em Suspensão empregando o sensor OLI na costa de Caravelas, Bahia, Brasil. GeoFocus, Revista Internacional de Ciencia y Tecnología de la Información Geográfica, 26, 121-137. http://dx.doi.org/10.21138/GF.674

good adjustment for 12/29/2014 $\left(\mathrm{RMSE}=5.89 ; \mathrm{RE}=19.2 \% ; \mathrm{R}^{2}=0.89 ; \mathrm{N}=14\right)$ and a reasonable adjustment for the image of 01/30/15 $2015\left(\mathrm{RMSE}=13.33 ; \mathrm{RE}=57 \% ; \mathrm{R}^{2}=0.5 ; \mathrm{N}=6\right)$. The model proved to be consistent. However, the monitoring of impacts resulting from dredging activities would be more effective after a new work outlined to consider the regional variability of TSS, including the plume generated by dredging.

Keywords: Abrolhos Marine National Park; Landsat 8; ocean color; coral reefs; impacts.

\section{Introdução}

Elevadas concentrações de sólidos em suspensão e nutrientes podem ocasionar impactos na produtividade primária devido à redução da penetração de luz na camada fótica, florescimento de cianobactérias e eutrofização (Zhang et al., 2016; Jaelani et al., 2016). O Total de Sólidos em Suspensão (TSS) presentes na zona costeira costumam refletir a estrutura geológica e a composição do continente ou bacia hidrográfica adjacente (IOCCG, 2000). O TSS desempenha um papel importante na gestão da qualidade da água, processos de erosão e deposição, produção primária, além de transporte de nutrientes, micropoluentes e metais pesados (Volpe et al., 2010; Hongyan, 2010).

A região da Foz do Rio Caravelas caracteriza-se pela ocorrência natural de elevados níveis de turbidez. Por outro lado, sua proximidade dos recifes coralinos do Arco Interno do Banco de Abrolhos (Leão, 1994), pode causar efeitos deletérios na biodiversidade recifal. A presença de afloramentos do Grupo Barreiras próximos à costa, a evolução da Planície Costeira em Caravelas durante o Quaternário e as mudanças no nível médio do mar, são fatores responsáveis por um expressivo aporte de sedimentos ao longo desta porção da costa baiana (Andrade, 2000). A ocupação inadequada da planície costeira na região é outro fator que pode agravar o incremento no aporte de sedimentos fluviais finos para o complexo recifal dos Abrolhos (Andrade \& Dominguez, 2002).

Imagens OLI, assim como sensores anteriores das plataformas Landsat, como o MSS, TM e $\mathrm{ETM}+$, têm se mostrado eficazes em estudos e monitoramentos de concentrações de TSS em zonas costeiras e oceânicas (Kerrigan \& Ali, 2020; Tu et al., 2018; Jaelani, 2016; Laili et al., 2015; Zhang et al., 2016; Slonecker, 2016; Mielke, 2014; Montanher et al., 2014). O L8, lançado em 2013, representa a continuidade da missão Landsat Data e está composto por dois sensores. O Operational Land Imager (OLI) que, além das bandas espectrais tradicionais, apresenta uma nova adaptada para observações da zona costeira e outra destinada à detecção de nuvens cirrus. Outro sensor a bordo do L8 é o Thermal Infrared Sensor (TIRS) capaz de obter dados termais. O sensor OLI fornece uma cobertura total da Terra com uma resolução espacial de 30 metros (visível, NIR, SWIR) e 15 metros (pancromática), enquanto a resolução do TIRS é de 100 metros (USGS, 2016). Não obstante, a resolução temporal das imagens L8 pode ser incrementada por meio de fusão com outros sensores como Sentinel-2 (Wang et al., 2017). Além disso, a família Landsat está em órbita desde a década de setenta, o que torna possível a obtenção de longas séries temporais (Qiu, 2019). 
Rocha Paulinha, P., Reuss Strenzel, GM. (2020). Um modelo empírico para estimativa do Total de Sólidos em Suspensão empregando o sensor OLI na costa de Caravelas, Bahia, Brasil. GeoFocus, Revista Internacional de Ciencia y Tecnología de la Información Geográfica, 26, 121-137. http://dx.doi.org/10.21138/GF.674

A estimativa de parâmetros referentes à qualidade da água, como a concentração de TSS e clorofila-a (Chl-a) através de imagens de satélite, depende da eficácia na correção atmosférica e parâmetros de recuperação de algoritmos de qualidade da água e cor do oceano (Laili et al.,2015; Gordon e Morel, 1983; Gordon, 1997). A remoção da influência da atmosfera em imagens na faixa espectral do visível é essencial para evitar superestimativas nas previsões de indicadores da qualidade da água, pois mais de $80 \%$ da luz que alcança o sensor orbital pode ter origem atmosférica (Morel, 1980).

Schettini (2010), Pereira (2010), Dominguez (2007) e Dominguez (2005) investigaram aspectos físico-químicos do estuário e da zona costeira de Caravelas, com o intuito de obter informações sobre áreas impactadas por sedimentos em suspensão. Entretanto, existe a necessidade de se ampliar as escalas espaciais e temporais de análise para um melhor entendimento dos impactos da dispersão do material particulado sobre os recifes coralinos do Arco Interno de Abrolhos. Através do sensoriamento remoto é possível gerar informações sobre os padrões de dispersão do material particulado e sua influência sobre a qualidade da água.

A área de interesse localiza-se na região costeira de Caravelas, Extremo Sul da Bahia (Figura 1), entre as coordenadas $39^{\circ} 17^{\prime} 16^{\prime \prime} \mathrm{S}, 1^{\circ} 55^{\prime} 34^{\prime \prime} \mathrm{O}$ e $-39^{\circ} 30^{\prime} 52^{\prime \prime} \mathrm{S}, 17^{\circ} 93^{\prime} 81^{\prime \prime O} \mathrm{O}$. A monocultura de eucalipto destinada a abastecer a indústria da celulose é uma das atividades econômicas mais importantes no Extremo Sul da Bahia. Para viabilizar o escoamento da biomassa desde áreas de cultivo até a fábrica da Aracruz em Barra do Riacho (ES), a Fibria Celulose construiu em 2003 um terminal portuário às margens do Rio Caravelas em Ponta de Areia, Caravelas, BA. Para manter seu funcionamento, têm sido necessárias dragagens periódicas para o aprofundamento do "Canal do Tomba", desde fevereiro de 2002. O descarte do material dragado é lançado sobre o fundo inconsolidado de uma área costeira próxima (Fig. 1).

Desde o início das atividades de dragagem, algumas comunidades costeiras da região, principalmente no município de Nova Viçosa, além de pescadores da Reserva Extrativista de Cassurubá (RESEX), relataram um aumento de sedimentos em suspensão, provocando prejuízos à produção pesqueira, maior dificuldade de navegação e influência negativa no turismo. Estes fatos levaram o Ministério Público do Estado da Bahia a instaurar um inquérito civil a fim de apurar eventuais danos ambientais causados pela Fibria Celulose na área de dragagem.

Este trabalho visa, portanto, contribuir para o entendimento da dinâmica sedimentar e distribuição espacial do material particulado em suspensão na costa do Extremo Sul da Bahia, propondo um modelo empírico para a estimativa de concentração de TSS com base em imagens OLI e dados obtidos em campo. 


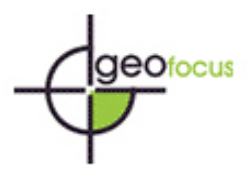

Rocha Paulinha, P., Reuss Strenzel, GM. (2020). Um modelo empírico para estimativa do Total de Sólidos em Suspensão empregando o sensor OLI na costa de Caravelas, Bahia, Brasil. GeoFocus, Revista Internacional de Ciencia y Tecnología de la Información Geográfica, 26, 121-137. http://dx.doi.org/10.21138/GF.674

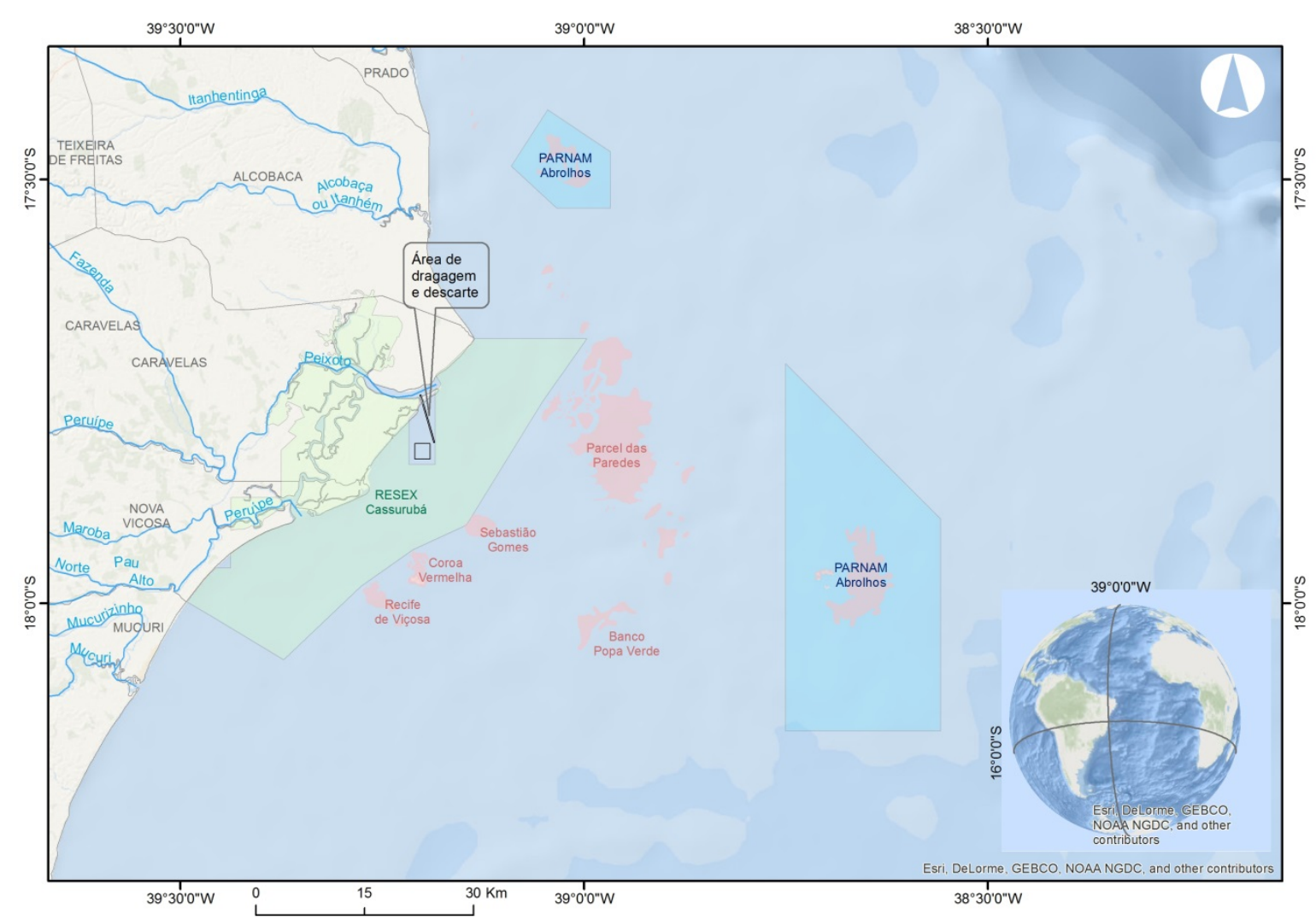

Figura 1: Localização da área de interesse.

\section{Materiais e Métodos}

\subsection{Dados de TSS obtidos in situ}

Os dados de TSS obtidos in situ foram recopilados do Relatório Técnico Integrado Consolidado, elaborado em agosto de 2015, pela Fibria Celulose S.A., atendendo a condicionante 2.8 da Licença de Operação da Dragagem do Canal do Tomba, Caravelas (BA), emitida pelo Instituto Brasileiro do Meio Ambiente e dos Recursos Naturais Renováveis (IBAMA), disponível em http://www.ibama.gov.br/licenciamento/. Os dados recopilados estão disponíveis em laudos produzidos por uma auditoria ambiental independente vinculada à Fundação Universitária de São Paulo (FUSP).

Os dados fazem parte de um esforço para o monitoramento da distribuição espacial da turbidez através da concentração do Total Sedimentos em Suspensão (mg/L) em três diferentes profundidades (superfície, meia água e fundo) em 26 estações dispostas ao longo de 5 perfis 


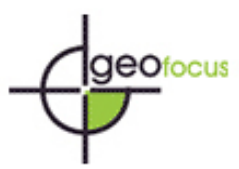

Rocha Paulinha, P., Reuss Strenzel, GM. (2020). Um modelo empírico para estimativa do Total de Sólidos em Suspensão empregando o sensor OLI na costa de Caravelas, Bahia, Brasil. GeoFocus, Revista Internacional de Ciencia y Tecnología de la Información Geográfica, 26, 121-137. http://dx.doi.org/10.21138/GF.674

transversais à linha de costa, durante os períodos de dragagem e pós-dragagem. As coordenadas dos pontos de coleta foram obtidas com DGPS. As amostras utilizadas na elaboração do modelo preditivo foram coletadas em superfície $(0,50 \mathrm{~cm}$ de profundidade) com garrafas de "Van Dorn" e refrigeradas em isopor até a entrega no laboratório para análise da concentração do TSS. O processamento para determinar o TSS foi realizado através do método SMEWW $22^{\circ}$ ED. 2012, 2540 D. Os resultados das análises foram disponibilizados em Laudos Laboratoriais da Campanha de 2014-2015, disponível em http://licenciamento.ibama.gov.br/.

Para criação do modelo foram empregados dados obtidos em 14 pontos amostrais no dia 29 de dezembro de 2014, além de dados obtidos em 6 pontos coletados no dia 29 de janeiro de 2015.

\subsection{Aquisição de Dados Oceanográficos}

Foram adquiridos dados meteorológicos e oceanográficos para a melhor compreensão da dinâmica do TSS na zona costeira de Caravelas. A precipitação acumulada, bem como velocidade e direção do vento nas últimas 36 horas antes de ambas as passagens do satélite foram recopilados da base de dados do Instituto Nacional de Meteorologia - INMET. (Tabela 1). Altura e direção do fluxo de maré (vazante ou enchente) foram estimados para os horários da passagem do satélite, a partir de previsões realizadas pela ferramenta $W W W$ Tide and Current Predictor, hospedado em http://tbone.biol.sc.edu/tide/ (Tabela 2).

\subsection{Processamento das imagens OLI}

Para elaboração de um modelo preditivo do TSS em superfície foram utilizados dados do sensor $O L I$, instalado a bordo do satélite Landsat 8 . As imagens foram adquiridas em duas datas distintas, conforme a coincidência com as datas de campanha de campo (29/12/2014 e 30/01/2015), no mecanismo de busca do Serviço Geológicos dos Estados Unidos United States Geological Survey - USGS, disponível em: http://earthexplorer.usgs.gov/.

Duas imagens foram adquiridas para realização do trabalho. A primeira, obtida em 29 de dezembro de 2014, foi empregada na elaboração do modelo de predição, pois a data de aquisição coincide com a coleta de dados em campo. Uma imagem alternativa obtida em 30 de janeiro de 2015, um dia após a coleta de 6 pontos de amostragem, foi utilizada na tentativa de viabilizar uma validação complementar do modelo. O processamento realizado sobre as imagens é descrito a seguir.

\subsubsection{Correção Atmosférica}

A correção atmosférica foi realizada empregando a extensão ATCOR2 (Atmospheric and Topographic Correction for Satellite Imagery) do software ERDAS IMAGINE 2014®. O ATCOR2 emprega um conjunto de modelos de transferência radiativa denominado MODTRAN (Moderate Resolution Atmospheric Transmission), que simulam a transmitância da atmosfera em condições climáticas e ângulos solares especificados pelo usuário, com a aplicando os resultados diretamente 


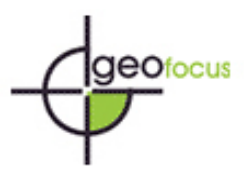

Rocha Paulinha, P., Reuss Strenzel, GM. (2020). Um modelo empírico para estimativa do Total de Sólidos em Suspensão empregando o sensor OLI na costa de Caravelas, Bahia, Brasil. GeoFocus, Revista Internacional de Ciencia y Tecnología de la Información Geográfica, 26, 121-137. http://dx.doi.org/10.21138/GF.674

sobre a imagem (RICHTER, 2000). Os dados sobre condições espectrais e de iluminação, necessárias à execução do modelo, foram obtidas diretamente do metadata da imagem. O modelo de transferência escolhido para simular as condições da atmosfera foi o "tropical marinho" e a visibilidade horizontal do dia foi estimada em $10 \mathrm{~km}$ pelo próprio ATCOR2.

\subsubsection{Eliminação de Nuvens}

A eliminação das nuvens nas imagens foi efetuada com base na banda BQA (controle de qualidade), conforme instruções disponíveis no manual do Landsat 8 (USGS, 2016). A banda BQA foi transformada numa máscara binária por reclassificação e multiplicada pelas imagens originais por meio da calculadora raster. Ambas operações foram efetuadas no software Idrisi 15.1®.

\subsubsection{Eliminação do Continente}

A eliminação do continente nas imagens foi efetuada pela aplicação de uma máscara binária elaborada por reclassificação do Índice da Diferença Normalizada de Água (Normalized Difference Water Index - NDWI) (MCFEETERS, 1996) (Equação 1). O resultado da equação é uma imagem com pixels negativos sobre o continente e positivos sobre o oceano. A imagem NDWI foi, em seguida, convertida em uma máscara binária por reclassificação, de modo que os pixels negativos foram transformados em zero e os positivos em um. A máscara binária foi, então, multiplicada às imagens originais com a calculadora raster. Estes procedimentos foram realizados com o software Idrisi $15.1{ }^{\circledR}$.

$$
N D W I=\frac{(B 3-B 5)}{(B 3+B 5)}
$$

Onde B3 corresponde à banda 3 do sensor OLI (verde) e B5 corresponde à banda 5 (infravermelho próximo).

\subsubsection{Transformação dos dados em Logaritmo Natural}

A eliminação do continente e nuvens resultou no isolamento dos pixels correspondentes ao oceano nas imagens, os quais foram transformados em logaritmo natural empregando com o Idrisi $15.1{ }^{\circledR}$. A absorção da radiação eletromagnética pela coluna d'água ocorre de maneira exponencial (GREEN, 2000). Assim, a transformação das bandas de ambas as imagens, bem como dos dados de TSS obtidos in situ em escala logarítmica possibilitou a linearização da relação entre as variáveis dependentes e explicativas no modelo.

\subsubsection{Análise de Componentes Principais S-Mode}

Uma análise exploratória preliminar foi realizada para investigar as relações existentes entre as variáveis explicativas (bandas da imagem de 29/12/2014) e a variável dependente (TSS). Este procedimento revelou que as bandas 2, 3 e 4 (azul: $452-512 \mathrm{~nm}$; verde: $533-590 \mathrm{~nm}$; e vermelho: 
Rocha Paulinha, P., Reuss Strenzel, GM. (2020). Um modelo empírico para estimativa do Total de Sólidos em Suspensão empregando o sensor OLI na costa de Caravelas, Bahia, Brasil. GeoFocus, Revista Internacional de Ciencia y Tecnología de la Información Geográfica, 26, 121-137. http://dx.doi.org/10.21138/GF.674

636-673 nm) apresentaram as melhores relações lineares com o TSS. No entanto, as três apresentaram forte mullticolinearidade, o que impede o uso do conjunto num modelo de regressão múltipla. Com o intuito de reduzir a redundância entre as variáveis explicativas, optou-se por realizar uma Analise de Componentes Principais Modo-S (PCA S-Mode), calculada a partir da matriz de covariância não estandardarizada das reflectâncias no topo da atmosfera registradas pelas bandas OLI 2, 3 e 4 log-transformadas, empregando o software Idrisi 15.1.

A PCA S-Mode, também denominada análise de Função Ortogonal Empírica (Empirical Orthogonal Function - EOF), considera cada pixel como um vetor (pixel-vetor) partindo da origem do espaço espectral formado pelo conjunto original de imagens. O resultado da análise é um novo conjunto de imagens ortogonais entre si, ordenadas pela quantidade de variância que cada uma explica em relação ao conjunto original (EASTMAN, 2006). Nos resultados do PCA S-Mode, os componentes propriamente ditos são apresentados em forma de tabelas, enquanto as imagens resultantes são as cargas dos componentes (components loadings) e representam a correlação entre cada pixel-vetor e o eigenvector. Os eigenvalues também são apresentados na forma de imagens, porém estas não foram empregadas na elaboração do modelo.

\subsection{Regressão}

O ajuste do modelo linear mais adequado para simulação do TSS a partir da imagem obtida em 29 de dezembro de 2014 foi realizado por meio do método dos Mínimos Quadrados empregando a ferramenta Ordinary Least Square $(O L S)$ do software ArcGis 10.3. As reflectâncias log-transformadas das bandas na faixa do visível da imagem OLI: $\operatorname{Ln}(\mathrm{R} 2), \operatorname{Ln}(\mathrm{R} 3), \operatorname{Ln}(\mathrm{R} 4)$, além das cargas da primeira e segunda componentes da PCA foram testadas como variáveis explicativas. O Critério de Informação de Akaike Corrigido (Corrected Akaike Information Criterion - AICc) foi empregado na escolha das variáveis mais adequadas para elaboração do modelo. Os modelos com menores valores de AICc foram considerados aqueles com melhor ajuste aos dados observados (Wagenmakers; Farrell, 2004).

$\mathrm{O}$ ajuste linear do modelo foi avaliado pelo coeficiente de determinação múltipla $\left(\mathrm{R}^{2}\right) \mathrm{e}$ coeficiente de determinação ajustado ( $\mathrm{R}^{2}$ Ajustado). $\mathrm{O}$ teste $\mathrm{T}$ foi empregado para avaliar se as variáveis explicativas empregadas na regressão eram estatisticamente significativas. A significância do conjunto de parâmetros empregados no modelo foi avaliada pela Estatística-F (Joint F-Stattistic) e pelo teste estatístico conjunto de Wald (Joint Wald Statistic) (Nelson, et al, 2006; Zhang et al., 2009; Garaba; Zielinski, 2015).

Adicionalmente a autocorrelação espacial dos resíduos resultantes da regressão foi investigada com auxílio da ferramenta Autocorrelation (Global Moran's I) do ArcGis 10.3, para corroborar as estimativas de significância das variáveis explicativas empregadas (Batty et al., 1996).

\subsection{Validação}

Os coeficientes de regressão do modelo que apresentaram o melhor ajuste foram empregados para gerar um mapa de TSS previsto para cada imagem OLI processada. Os mapas 


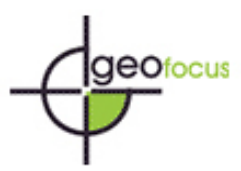

Rocha Paulinha, P., Reuss Strenzel, GM. (2020). Um modelo empírico para estimativa do Total de Sólidos em Suspensão empregando o sensor OLI na costa de Caravelas, Bahia, Brasil. GeoFocus, Revista Internacional de Ciencia y Tecnología de la Información Geográfica, 26, 121-137. http://dx.doi.org/10.21138/GF.674

preditivos foram gerados com a calculadora raster do software Idrisi $15.1{ }^{\circledR}$, substituindo-se a variável independente da equação da reta pelas imagens OLI.

A validação do modelo foi realizada por meio da comparação entre os valores previstos de TSS nos mapas e os valores observados nas amostragens, mediante o cálculo do Desvio MédioQuadrático (Root Mean Square Error-RMSE) (Equação 2), do Erro Relativo (Relative Error - RE) (Equação 3) e do Coeficiente de Determinação $\left(\mathrm{R}^{2}\right)$. Onde o RMS reflete a dispersão da reflectância do sensoriamento remoto bem como as concentrações de parâmetros de qualidade da água, RE representa a incerteza associada a distribuição pelo satélite e $\mathrm{R}^{2}$ a relação existente entre a reflectância medida in situ e a as concentrações de TSS previstas pelo modelo.

$$
\begin{aligned}
& R M S E=\frac{\sqrt{\sum_{J=1}^{N}\left({\text { xest } \left.i, i-x_{\text {meas }}, i\right)^{2}}^{2}\right.}}{N} \\
& R E=\frac{1}{N} \sum_{j=1}^{N}\left|\frac{x_{\text {est } i, i-x_{\text {meas }, i}}}{x_{\text {meas }}}\right| 100 \%
\end{aligned}
$$

Onde para RMSE e RE: Xmeas, $i$ e Xesti, $i$ são, respectivamente, os valores de TSS medidos e estimados, e $\mathrm{N}$ é o número de amostras.

\section{Resultados}

\subsection{Dados oceanográficos e meteorológicos}

Uma base de dados (Tabelas 1 e 2), incluindo os dados oceanográficos, imagens OLI transformadas em logaritmo e dados de TSS coletados in situ (Fig. 2).

Tabela 1: Maré, altura da maré, fase da lua no dia da passagem do satélite e precipitação acumulada nas últimas 72 horas antes da passagem do satélite.

\begin{tabular}{cccccc}
\hline Data & Maré & $\begin{array}{c}\text { Altura da } \\
\text { maré }(\mathbf{m})\end{array}$ & $\begin{array}{c}\text { Fase da } \\
\text { Lua }\end{array}$ & $\begin{array}{c}\text { Precipitação } \\
\text { Acumulada } \mathbf{7 2} \mathbf{h} \\
\mathbf{( m m}\end{array}$ & $\begin{array}{c}\text { Precipitação } \\
\text { Acumulada mês } \\
(\mathbf{m m})\end{array}$ \\
\hline $29 / 12 / 2014$ & Preamar & 1,45 & Crescente & 0,1 & 58,8 \\
$30 / 01 / 2015$ & Enchente & 1,07 & Crescente & 2 & 16 \\
\hline
\end{tabular}


Rocha Paulinha, P., Reuss Strenzel, GM. (2020). Um modelo empírico para estimativa do Total de Sólidos em Suspensão empregando o sensor OLI na costa de Caravelas, Bahia, Brasil. GeoFocus, Revista Internacional de Ciencia y Tecnología de la Información Geográfica, 26, 121-137. http://dx.doi.org/10.21138/GF.674

Tabela 2: Velocidade e direção do Vento nas últimas $72 \mathrm{~h}$ antes da passagem do satélite

\begin{tabular}{cccccccc}
\hline Data & Hora & $\begin{array}{c}\text { Velocida do } \\
\text { Vento }(\mathbf{k m} / \mathbf{h})\end{array}$ & $\begin{array}{c}\text { Direcão } \\
\text { do Vento }\end{array}$ & Data & Hora & $\begin{array}{c}\text { Velocida de } \\
\text { Vento }(\mathbf{k m} / \mathbf{h})\end{array}$ & $\begin{array}{c}\text { Direcão } \\
\text { do Vento }\end{array}$ \\
\hline $27 / 12 / 2014$ & $00: 00$ & 0 & $\mathrm{C}$ & $28 / 01 / 2015$ & $00: 00$ & 0 & $\mathrm{C}$ \\
$27 / 12 / 2014$ & $12: 00$ & 5,5 & $\mathrm{SE}$ & $28 / 01 / 2015$ & $12: 00$ & 5,5 & $\mathrm{NE}$ \\
$27 / 12 / 2014$ & $18: 00$ & 5,5 & $\mathrm{SE}$ & $28 / 01 / 2015$ & $18: 00$ & 11,1 & $\mathrm{NE}$ \\
$28 / 12 / 2014$ & $00: 00$ & 0 & $\mathrm{C}$ & $29 / 01 / 2015$ & $00: 00$ & 0 & $\mathrm{C}$ \\
$28 / 12 / 2014$ & $12: 00$ & 11,1 & $\mathrm{NE}$ & $29 / 01 / 2015$ & $12: 00$ & 0 & $\mathrm{C}$ \\
$28 / 12 / 2014$ & $18: 00$ & 11,1 & $\mathrm{NE}$ & $29 / 01 / 2015$ & $18: 00$ & 14,8 & $\mathrm{E}$ \\
$29 / 12 / 2014$ & $00: 00$ & 5,5 & $\mathrm{NE}$ & $30 / 01 / 2015$ & $00: 00$ & 0 & $\mathrm{C}$ \\
$29 / 12 / 2014$ & $12: 00$ & 12,9 & $\mathrm{NE}$ & $30 / 01 / 2015$ & $12: 00$ & 9,2 & $\mathrm{NE}$ \\
\hline Média & $\mathbf{6 . 5}$ & & & & $\mathbf{5 . 1}$ & \\
Desvio Padrão & $\mathbf{4 . 9}$ & & & & $\mathbf{6 . 0}$ & \\
\hline
\end{tabular}

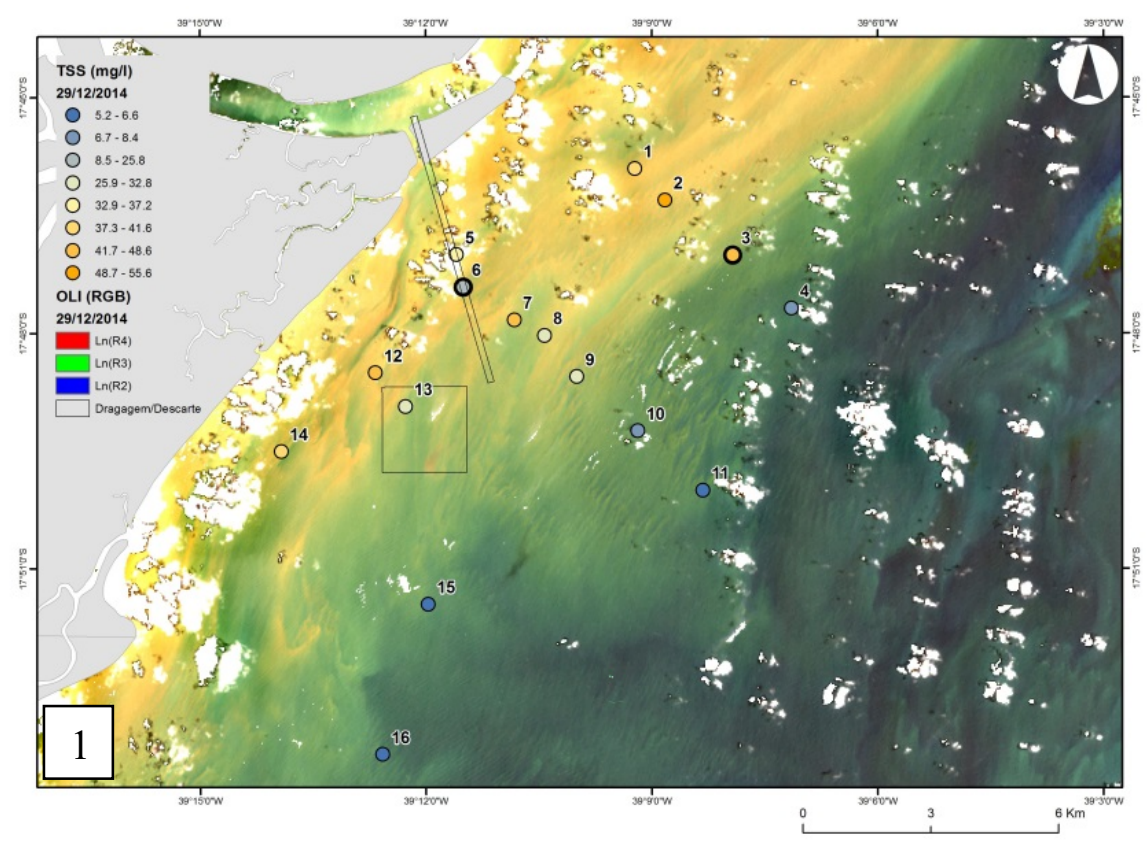


Rocha Paulinha, P., Reuss Strenzel, GM. (2020). Um modelo empírico para estimativa do Total de Sólidos em Suspensão empregando o sensor OLI na costa de Caravelas, Bahia, Brasil. GeoFocus, Revista Internacional de Ciencia y Tecnología de la Información Geográfica, 26, 121-137. http://dx.doi.org/10.21138/GF.674

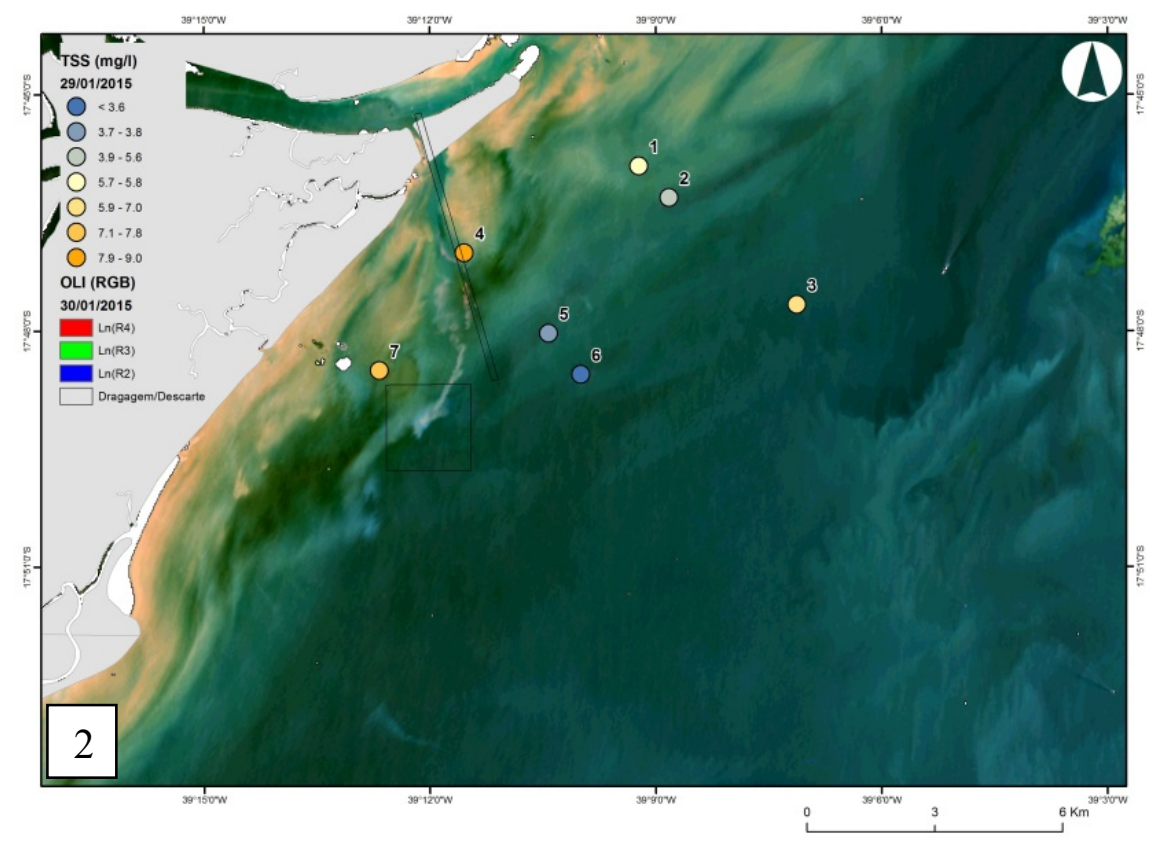

Fig. 2. Valores de TSS amostrados em 29/12/2014 sobrepostos a uma composição em cor verdadeira empregando a reflectância no topo da atmosfera registrada nas bandas OLI 2, 3 e 4 logtransformadas na mesma data. Os pontos 3 e 6 foram identificados como outliers (1). Valores de TSS amostrados em 29/01/2015 a uma composição em cor verdadeira empregando a reflectância no topo da atmosfera registrada nas bandas OLI 2, 3 e 4 log-transformadas no dia 30/01/2015 (2).

\subsection{Modelo e mapas preditivos de TSS}

Após a análise exploratória realizada, verificou-se que o modelo de regressão com menor valor de AICc $(-0,322)$ e, portanto, como melhor ajuste foi obtido utilizando somente a carga da primeira componente da PCA S-Mode $\left(\mathrm{PCA}_{1}\right)$ como variável explicativa. A porcentagem de variância explicada pelo primeiro e segundo componentes da imagem de 29/12/2014 foi de 76,7 \% e 32,3\%, respectivamente. Já os dois primeiros componentes da imagem de 30/01/2015 explicaram $67,7 \%$ e $32,3 \%$. A análise exploratória revelou também a presença de dois outliers na imagem de 29/12/2014 (Fig. 2) os quais foram eliminados do modelo de regressão.

Os valores de $\mathrm{R}^{2}$ e $\mathrm{R}^{2}$ ajustado obtidos pela regressão por mínimos quadrados entre as cargas da primeira componente PCA e o valores de TSS log-transformados foram ambos de 0,94 . O teste $\mathrm{T}$ revelou que os coeficientes de regressão foram altamente significativos ( $\mathrm{p}$-valores $<0,001$ ), com erro padrão de 0,074 e a estatística-T de 32,78 para o intercept e erro padrão de 0,093 e estatística-T de $-14,18$ para o coeficiente da variável independente. Os p-valores para os testes de Wald e estatística-F mostraram-se altamente significativos ( $p$-valor $<0,001$ ). Além disso, o teste de autocorrelação espacial dos resíduos revelou que seu padrão de distribuição é aleatório, uma vez que o z-score resultante foi igual a 0,65 . 
Rocha Paulinha, P., Reuss Strenzel, GM. (2020). Um modelo empírico para estimativa do Total de Sólidos em Suspensão empregando o sensor OLI na costa de Caravelas, Bahia, Brasil. GeoFocus, Revista Internacional de Ciencia y Tecnología de la Información Geográfica, 26, 121-137. http://dx.doi.org/10.21138/GF.674

Os coeficientes de regressão linear que compõem o modelo empírico (Equação 4) de foram usados para elaboração dos mapas de previsão de TSS (Fig. 4).

$$
\operatorname{Ln}(T S S)=2.428-1,321\left(\mathrm{PCA}_{1}\right)
$$

Onde $\mathrm{PCA}_{1}$ representa as cargas da primeira componente da PCA S-Mode efetuada com $\mathrm{Ln}(\mathrm{R} 2), \mathrm{Ln}(\mathrm{R} 3)$ e $\mathrm{Ln}(\mathrm{R} 4)$, sendo que R representa a reflectância no topo da atmosfera.

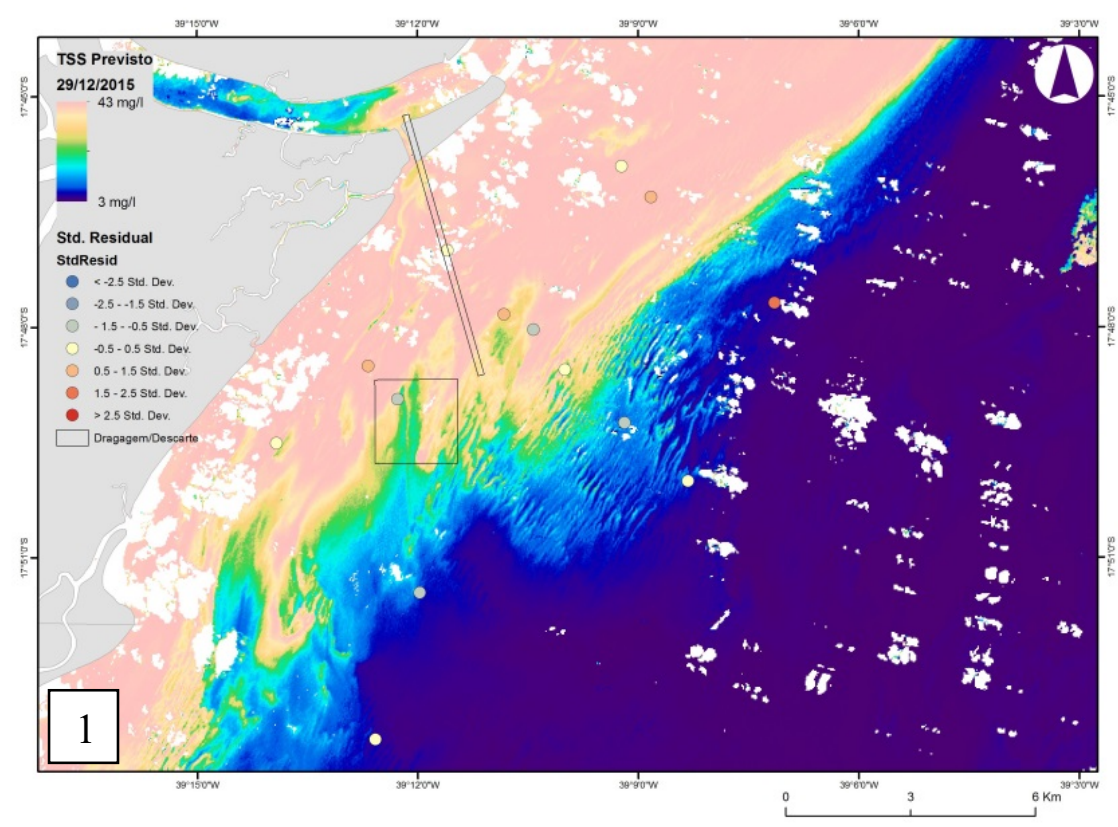


Rocha Paulinha, P., Reuss Strenzel, GM. (2020). Um modelo empírico para estimativa do Total de Sólidos em Suspensão empregando o sensor OLI na costa de Caravelas, Bahia, Brasil. GeoFocus, Revista Internacional de Ciencia y Tecnología de la Información Geográfica, 26, 121-137. http://dx.doi.org/10.21138/GF.674

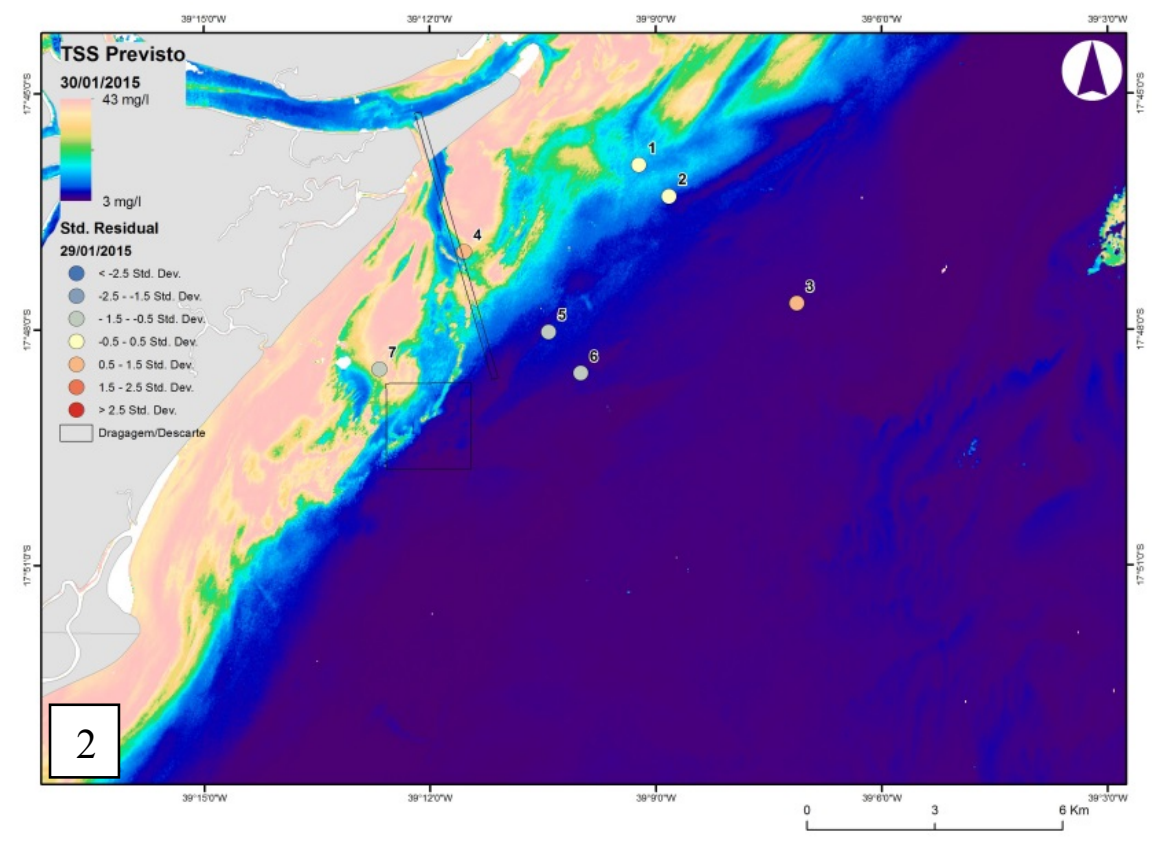

Fig. 4. Mapa de previsão do TSS para o dia 29/12/2014 sobreposto aos valores dos resíduos padronizados em cada ponto de amostragem (1). Mapa de previsão do TSS para o dia 30/01/2015 sobreposto aos valores dos resíduos padronizados em cada ponto de amostragem (2).

\subsection{Validação}

A comparação entre o TSS previsto em ambos os mapas comparados com os valores amostrados revelaram um bom ajuste para a imagem do dia 29/12/2014, com $\mathrm{RMSE}=5,89$; $\mathrm{RE}=$ $19,2 \% ; \mathrm{R}^{2}=0,89 ; \mathrm{N}=14$. Por outro lado, a imagem obtida em 30/01/2015 demonstrou um menor ajuste, com $\mathrm{RMSE}=13,33 \mathrm{mg} / \mathrm{l} ; \mathrm{RE}=57 \% ; \mathrm{R}^{2}=0,5 ; \mathrm{N}=6$ (Fig. 5). 


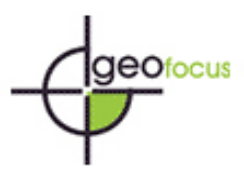

Rocha Paulinha, P., Reuss Strenzel, GM. (2020). Um modelo empírico para estimativa do Total de Sólidos em Suspensão empregando o sensor OLI na costa de Caravelas, Bahia, Brasil. GeoFocus, Revista Internacional de Ciencia y Tecnología de la Información Geográfica, 26, 121-137. http://dx.doi.org/10.21138/GF.674

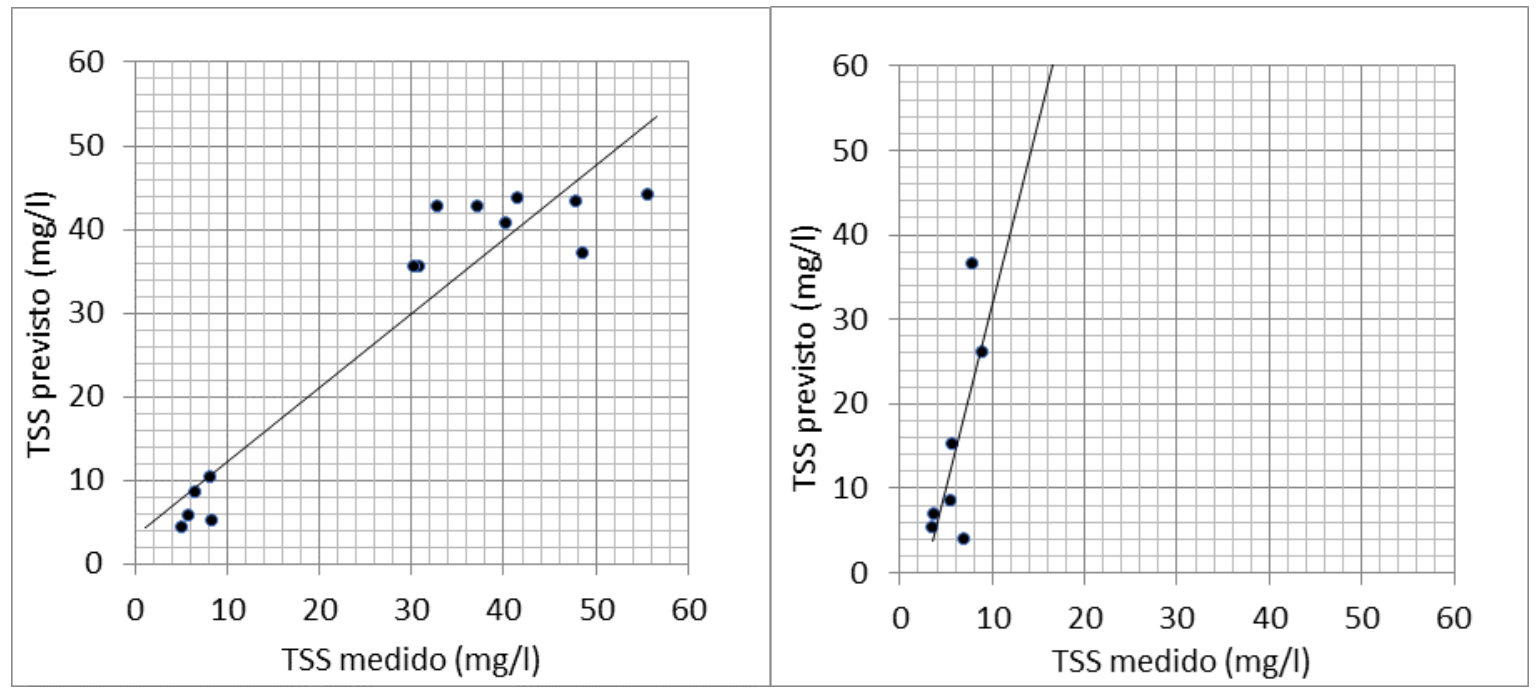

Fig. 5. Diagrama de dispersão comparando as concentrações de TSS medidos in situ previstas pelo modelo no dia 29/12/2014 (esquerda) e 30/01/2015 (direita).

\section{Discussão}

O modelo de empírico de previsão de TSS obtido foi considerado aceitável para estimar o sedimento em suspensão na costa de Caravelas. Os coeficientes de determinação ( $\mathrm{R}^{2}$ e $\mathrm{R}^{2}$ ajustado) chegaram próximos a seu valor máximo e os testes de significância de Waden e estatística-F indicaram que o conjunto das variáveis aplicadas no modelo é consistente. Além disso, o teste de autocorrelação espacial efetuado com os resíduos revelou que estes apresentam distribuição aleatória. Esta análise indica que a estratégia adotada em empregar o resultado de uma PCA empregando a reflectância no topo da atmosfera registrada pelas bandas no visível do sensor OLI log-transformada, foi coerente com o intuito de agrupar as variáveis explicativas mais significativas para simulação da distribuição do TSS.

A única banda visível do sensor OLI não incluída na regressão foi Coastal Blue (Banda 1: 435-451 nm). Trata-se de uma nova faixa espectral que não fazia parte dos antigos sensores da missão Landsat (MSS e TM). Essa banda representa um grande avanço em estudos da zona costeira, porém, durante a análise exploratória verificou-se que sua introdução no modelo causava uma diminuição nos parâmetros de qualidade do modelo analisados. Isso se deve, possivelmente, ao fato dessa faixa espectral ser fortemente contaminada pela atmosfera, conforme verificado durante seu processamento. Ao mesmo tempo, por apresentar um menor coeficiente de extinção na água, espera-se uma maior contaminação causada pelo fundo nas zonas mais rasas (GREEN et al., 2000).

O processo de validação revelou que a acurácia do mapa preditivo do TSS elaborado para o dia 29/12/2014 foi aceitável. Um modelo empírico semelhante, elaborado por Jaelani et al. (2016) a partir da razão entre as bandas 2 e 3 do sensor OLI em águas da Indonésia para estimativas de TSS, 
Rocha Paulinha, P., Reuss Strenzel, GM. (2020). Um modelo empírico para estimativa do Total de Sólidos em Suspensão empregando o sensor OLI na costa de Caravelas, Bahia, Brasil. GeoFocus, Revista Internacional de Ciencia y Tecnología de la Información Geográfica, 26, 121-137. http://dx.doi.org/10.21138/GF.674

apresentou $\mathrm{RMSE}=2,96 ; \mathrm{RE}=14,82 \% ; \mathrm{R}^{2}=0,34 ; \mathrm{N}=6$. De maneira geral, os erros médio e relativo foram de ordem semelhante, enquanto o coeficiente de determinação foi inferior. Por outro lado, acurácia do mapa elaborado a partir da imagem de 30/01/2015 foi bastante reduzida, quando comparado aos demais. Isso se deve, principalmente, à defasagem de um dia entre as coletas de TSS e a passagem do satélite. A análise desta imagem no modo truecolor revela que, possivelmente, os dados tomados in situ próximos à linha de costa estejam subestimando o TSS (Fig. 2). Com isso, há uma tendência das predições feitas para esse dia superestimarem os dados obtidos na amostragem. Ainda assim, o coeficiente de determinação foi superior ao obtido por Jaelani et al. (2016).

Tanto as composições em cor verdadeira quanto os mapas de preditivos de TSS e os dados obtidos in situ, mostram que a concentração de sedimento em 29/12/2014 foi bastante superior quando comparada ao dia 30/01/2015. Os dados oceanográficos e meteorológicos recopilados indicam que as condições climáticas eram semelhantes em ambas as passagens do satélite. A precipitação acumulada nos três dias anteriores é praticamente nula, apesar dos registros para o mês de dezembro indicarem uma precipitação cerca de três vezes superior àquela registrada em janeiro. No entanto, observando-se ambas as imagens, é possível perceber que a origem do sedimento em suspensão não se encontra no interior do estuário do Caravelas, que exibe águas relativamente limpas, e sim na plataforma interna. Apesar dos dados referentes à intensidade do vento sugerirem períodos relativamente calmos antecedendo ambas as imagens, a formação de nuvens observada na imagem do dia 29/12/2014 sugere a presença de vento de moderado a forte proveniente do quadrante leste no momento da passagem do satélite, o que pode explicar o elevado teor de sedimento em suspensão.

Uma característica marcante da costa de Caravelas, observável em todos os dados disponíveis é a presença de dois tipos de água com concentrações de sólidos em suspensão muito distintas. O primeiro tipo, que pode ser considerado "água costeira", ocupa uma faixa mais próxima à linha de costa e caracteriza-se por teores de TSS variando entre cerca de 25 e $56 \mathrm{mg} / \mathrm{l}$. O segundo, que pode ser considerado "água de plataforma", apresenta teores abaixo de $8 \mathrm{mg} / \mathrm{l}$ de TSS. A imagem de 30/01/2015 mostra claramente essa característica, porém os dados obtidos nas amostragens do dia anterior não revelam essa tendência.

Uma questão importante, que motivou a realização deste trabalho, é a capacidade dos mapas preditivos de TSS servirem como ferramenta no monitoramento da pluma gerada pelas atividades de dragagem do acesso do Canal do Tomba promovidas pela Fibria SA. Na imagem de 29/12/2014 a pluma é bastante reduzida e confunde-se com a elevada concentração natural de TSS das "águas costeiras". Segundo o Relatório Técnico elaborado pela Fibria SA, as atividades de dragagem deste ano, tiveram início nestes dias, o que pode ajudar a explicar o reduzido tamanho da pluma. Por outro lado, na imagem de 30/01/2015, a pluma gerada pela dragagem e pelo descarte é bastante evidente. O fato da "água costeira" estar ocupando uma zona mais estreita nessa data, além do maior tempo decorrido desde o início das dragagens podem, em conjunto, explicar esse fato (Fig. 2).

O mapa preditivo de TSS do dia 30/01/2015 evidencia a ocorrência da pluma sedimentar gerada pela atividade de dragagem, sem que nenhum ponto de amostragem tivesse sido realizado sobre ela. Para melhorar a capacidade dos mapas de predição do TSS evidenciarem a pluma de 


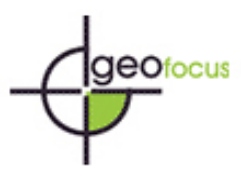

Rocha Paulinha, P., Reuss Strenzel, GM. (2020). Um modelo empírico para estimativa do Total de Sólidos em Suspensão empregando o sensor OLI na costa de Caravelas, Bahia, Brasil. GeoFocus, Revista Internacional de Ciencia y Tecnología de la Información Geográfica, 26, 121-137. http://dx.doi.org/10.21138/GF.674

dragagem, assim como os diferentes tipos de água, é importante a realização de um desenho amostral estratificado, capaz de representar a variabilidade existente em cada categoria.

\section{Discussão}

A estratégia adotada para elaboração do modelo empírico, empregando as cargas do primeiro componente da análise de componentes principais (PCA S-Mode) das bandas 2, 3 e 4 do sensor OLI mostrou-se viável para previsão do TSS na costa do Extremo Sul da Bahia. No entanto, para efetuar um monitoramento efetivo dos impactos decorrentes das atividades de dragagem do Canal do Tomba, é recomendável a aquisição de novos dados de campo, segundo um delineamento amostral capaz de apreender a variabilidade do TSS existente entre a água costeira, a água de plataforma e a pluma gerada pela atividade de dragagem em sincronia com a passagem do satélite.

\section{Referências bibliográficas}

Andrade A.C.S. Evolução quaternária da planície Costeira de Caravelas - extremo Sul do Estado da Bahia. 2000, 162 p. Tese de Doutorado, Instituto de Geociências da Universidade Federal da Bahia, 2000

Andrade ACS \& Dominguez JML. 2002. Informações Geológico Geomorfológicas como Subsídios à Análise Ambiental: o Exemplo da Planície Costeira de Caravelas - Bahia. Boletim Paranaense de Geociências,51: 9-17, 2002.

Batty, M.; Mesev, T. V.; Longley, P.; RS-GIS: Spatial Distributions from Remote Imagery. In: Longley, P and Batty, M, (eds.) Spatial Analysis: GIS in a Modelling Environment. Cambridge, UK. P. 123 - 148. 1996.

Banco Nacional de Dados OCeanográficos - BNDO. Disponível em: < http://www.mar.mil.br/dhn/chm/oceanografia/acesso_dados_produtos.html $>$ Acessado em 30/11/2016.

Dominguez J.M.L. et al. Sandy beaches of the eastern/ northeastern coast of Brazil: geographic variability and coastal erosion. In: SIMPÓSIO BRASILEIRO SOBRE PRAIAS ARENOSAS: Morfodin., Ecol., Riscos e Gestão, Itajaí, 2000.

Eastman, J.R Idrisi Manual, Clark University, Worcester. 2006.

Garaba, S. P. Zielinski, O. An assessment of water quality monitoring tools in an estuarine system, Remote Sensing Applications: Society and Environment. V. 2, P. 1-10. 2015.

Green, E. P., Mumby, P.J., Edwards, A. J., Clarck, C. D., (Ed. A. J. Edwards). Remote Sensing Handbook for Tropical Coastal Management. Coastal Management Sourcebooks 3, UNESCO, Paris. P. 316. 2000. 
Rocha Paulinha, P., Reuss Strenzel, GM. (2020). Um modelo empírico para estimativa do Total de Sólidos em Suspensão empregando o sensor OLI na costa de Caravelas, Bahia, Brasil. GeoFocus, Revista Internacional de Ciencia y Tecnología de la Información Geográfica, 26, 121-137. http://dx.doi.org/10.21138/GF.674

Gordon, H. R.; Morel, A. Remote assessment of ocean color for interpretation of satellite visible imagery: a review. In: Barber, Rt; Mooers, N.K.; Bowman, M. J.; Zeitzschel, B. (Ed.) Lecture notes on coastal and estuarine studies. New York: Springer- Verlag, 1983.

Hongyan, X; Yuanzhi, Z. Total suspended matter observation in the Pearl River estuary from in situ and MERIS data. Springer Science+Business Media B.V. 2010

IOCCG (2000). Remote Sensing of Ocean Colour in Coastal, and Other Optically-Complex, Waters. Sathyendranath, S. (ed.), Reports of the International Ocean-Colour Coordinating Group, No. 3, IOCCG, Dartmouth, Canada.

INMET - INSTITUTO NACIONAL DE METEOROLOGIA. Disponível em: < http://www.inmet.gov.br/portal// >. Acessado em 05/11/2016.

Jaelani, L. M.; Kurniadin, N. Empirical Algorithm Modeling for Estimating Total Suspended Solid Concentration Using In-situ Data and Atmospheric Corrected Landsat 8, Case Study: Gili Iyang's Waters. The 2nd Internasional Seminar on Science and Technology (ISST) for Sustainable Infrastructure Empowering Research and Technology for Sustainable Infrastructure - 2nd August 2016

Jaelani, L. M.; Limehuwey, R.; Kurniadin, N.; Pamungkas, A.; Koennhardono, E. S.; Sulisetyono, A. Estimation of TSS and Chl-a Concentration from Landsat 8-OLI: The Effect of Atmosphere and Retrieval Algorithm. The Journal for Technology and Science. V. 27. N. 1.2016

Laili, N.; Arafah L. M.; Jaelani, L. M.; Subehi, L.; Koenhadono, E. S.; Sulisetyono, A. Developement of water quality parameter retrieval algorithms for estimation total suspended solids and chlorophill-a concentration using landsat- 8 imagery at poteran island water. SPRS Annals of the Photogrammetry, Remote Sensing and Spatial Information Sciences, Volume II-2/W2, 2015 Joint International Geoinformation Conference 2015, 28-30 October 2015, Kuala Lumpur, Malaysia

Kerrigan, K.; Ali, K.A. Application of Landsat 8 OLI for monitoring the coastal waters of the US Virgin Islands. International Journal of Remote Sensing, V. 41, N. 15, pp.5743-5769, 2020.

Leão, Z. M. N. Threats to coral reefs environments. In: Corals of southern Bahia. Ed. Hetzel B. and Castro, C.B. Nova Fronteira, Rio de Janeiro, 1994

McFeeters, S.K. The use of the Normalized Difference Water Index (NDWI) in the delineation of open water features. International Journal of Remote Sensing, v.17, n.7, p.1425-1432, 1996.

Mielke, C.; Boesche, N. K.; Rogass, C.; Kaufmann, H.; Gauuert, C.; WIT, M. Spaceborne Mine Waste Mineralogy Monitoring in South Africa, Applications for Modern Push-Broom Missions: Hyperion/OLI and EnMAP/Sentinel-2. Remote Sensing. V. 6 (2014) 6790-6816

Montanher, O.C., Novo, E.M., Barbosa, C.C., Rennó, C.D. and Silva, T.S. Empirical models for estimating the suspended sediment concentration in Amazonian white water rivers using Landsat 5/TM. International Journal of Applied Earth Observation and Geoinformation, V. 29, pp.67-77, 2014. 


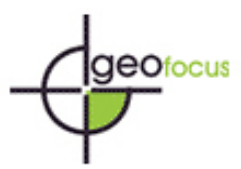

Rocha Paulinha, P., Reuss Strenzel, GM. (2020). Um modelo empírico para estimativa do Total de Sólidos em Suspensão empregando o sensor OLI na costa de Caravelas, Bahia, Brasil. GeoFocus, Revista Internacional de Ciencia y Tecnología de la Información Geográfica, 26, 121-137. http://dx.doi.org/10.21138/GF.674

Morel, A. In-water and remote measurements of ocean colour. Boundary Layer Meteorology. V.18, p. $177-201,1980$

Nascimento D. M. C.; Dominguez J. M. L. 2005. O mapa de vulnerabilidade ambiental como subsídio à gestão da zona costeira: o exemplo da planície costeira de Belmonte-BA. In: Congresso Brasileiro de Cartografia, 22, Anais, p.1-6.

National Oceanic and Atmospheric Administration - NOAA. Disponível em: < https://tidesandcurrents.noaa.gov/curr pred.html $>$ Acessado em: 30/11/2016

Nelson S. A. C.; Spence Cheruvelil, K.; Soranno, P. A. Satellite remote sensing of freshwater macrophytes and the influence of water clarity. Aquatic Botany, V. 85, N. 4, P. 289-298. 2006

Pereira, M. D., Siegle, E., Miranda, L. B., Schettini, C. A. F., 2010. Hidrodinâmica e transporte de material particulado em suspensão sazonal em um estuário dominado por maré: estuário de Caravelas (BA),_Revista Brasileira de Geofisica 28(3), 427-444.

Qiu, S., Lin, Y., Shang, R., Zhang, J., Ma, L. and Zhu, Z. Making Landsat time series consistent: evaluating and improving Landsat analysis ready data. Remote Sensing, V. 11, N. 1, p. 51, 2019.

Richter, R. Atmosferic correction algorithm for flat terrain: ATCOR2. Geosystems, 208 p. (2000).

Schettini, C. A. F.; Miranda, L. B. Circulation and suspended particulate matter transport in a tidally dominated estuary: Caravelas estuary, Bahia, Brazil. Brazilian Journal of Oceanography, v.58, n.1, p.1-11, 2010

Slonecker, E. T.; Jones, D. K.; Pellerin, B. A. The new landsat 8 potential for remote sensing of colored dissolved organic matter (CDOM). Marine Pollution Bulletin, V.107, P. 518-52, 2016.

Tu, M.C., Smith, P. and Filippi, A.M. Hybrid forward-selection method-based water-quality estimation via combining Landsat TM, ETM+, and OLI/TIRS images and ancillary environmental data. Plos one, V. 13, N. 7, p.e0201255, 2018.

USGS 2016. Landsat 8 (L8) Data Users Handbook Version 2.0, March, 2016. Department of the Interior - U.S. Geological Survey - NASA. Disponível em: < https://landsat.usgs.gov/landsat-8-18data-users-handbook $>$. Acesso em 05/11/2016.

Volpe, V; Marani, M.; Silvestre; S. Remote sensing retrieval of suspended sediment concentration in shallow waters. Remote Sensing of Environment, V. 115, P. 44-54. 2011.

Wagenmakers, E. J.; Farrell, S. AIC model selection using Akaike weights. Psychonomic Bulletin \& Review. V. 11, N. 1, P. 192-196. 2004.

Zhang, X.; Tang, D.; Li, Z. Zhang, F. The effects of wind and rainfall on suspended sediment concentration related to the 2004 Indian Ocean tsunami, Marine Pollution Bulletin, V. 58, N. 9, P. 1367-1373. 2009.

Zhang, Y.; Zhang, Y.; Shi, K.; Zha, Y.; Zhou, Y.; Liu, M. A Landsat 8 OLI-Based, Semianalytical Model for Estimating the Total Suspended Matter Concentration in the Slightly Turbid Xin'anjiang Reservoir (China). IEEE Journal of Selected Topics in Applied Earth Observations and Remote Sensing, V. 9. N. 1. P. 398-413. 2016. 
